\section{Cutoff Scores, Cutting Scores}

Sandra Banks

Department of Psychiatry, Allegheny General Hospital, Pittsburgh, PA, USA

\section{Synonyms}

Cut scores

\section{Definition}

Cutoff scores, also referred to as cutting scores, are scores that differentiate the levels of performance.

\section{Current Knowledge}

The reliability of a measure is an essential factor to consider when using cutoff scores. Nunnally and Bernstein (1994) suggest that a test must have an internal consistency coefficient of at least 0.90 , and ideally above 0.95 , to designate a particular score as a diagnostic cutoff point, although this might be a high standard. For instance, if a child is demonstrating a learning disorder, the tests used to assess the disorder must be reliably able to measure the particular abilities, taking into consideration the span of the standard error, to place an education-altering label on that child. If such standards are not followed, then clinicians run the risk of basing a decision on a "false-positive" (the discovery of a limitation when none exists in actuality) or a "false-negative" (the missing of a limitation when one truly exists).

To consider an example, a cutoff score may be the score differentiating between intact performance and impairment on a particular test. To speak in terms of diagnosing cognitive impairment, in a normal distribution of scores, a cutoff score would be any score below $95 \%$ which is obtained by the intact group within a particular standardization sample.

Clinicians may adjust cutoff scores because of individual or cultural variables that render the reliability of a test nonapplicable to the individual or sample being assessed. Otherwise, the clinician could run the risk of pathologizing an individual who does not truly exhibit the pathology.

\section{References and Reading}

Nunnally, J. C., \& Bernstein, I. H. (1994). Psychometric theory (3rd ed.). New York: McGraw-Hill. 